\title{
Lower urinary tract symptoms impair the quality of life in maintenance hemodialysis patients
}

\author{
Tadasuke Ando ${ }^{1,2^{*}}$, Takahisa Kamegawa ${ }^{3}$, Toshitaka Shin ${ }^{1}$ and Hiromitsu Mimata ${ }^{1}$
}

\begin{abstract}
Background: With aging, and as the duration of their dependence on dialysis becomes longer, maintenance hemodialysis patients experience decreased urine volume and lose the need to urinate. It is not known whether lower urinary tract symptoms (LUTS) affect the quality of life (QOL) in maintenance hemodialysis patients. We report an observational study that used validated questionnaires to evaluate LUTS and the influence of LUTS on QOL in a group of maintenance hemodialysis patients.
\end{abstract}

Methods: Stable patients who were on maintenance hemodialysis were surveyed with the Overactive Bladder Symptom Score and International Prostate Symptom Score, as the disease-specific validated questionnaires for overactive bladder and benign prostatic hypertrophy, to assess the LUTS and with King's Health Questionnaire to assess the influence of LUTS on QOL.

Results: This study included 131 patients with a mean age and hemodialysis duration of 69.1 years and 98.1 months. The disease-specific validated questionnaires found that 20 patients of them experienced LUTS. The mean QOL score of King's Health Questionnaire was significantly impaired $(p<0.01)$, and hemodialysis duration was significantly shorter $(p<0.01)$ in the LUTS patients than in the non-LUTS patients. Single and multiple regression analysis showed that LUTS was independently associated with the QOL score of King's Health Questionnaire (correlation coefficient 0.292; 95\% confidence interval 20.36-89.47; $p<0.01$ ).

Conclusions: LUTS exists in maintenance hemodialysis patients and impairs the QOL of them assessed with the validated questionnaires. Urological interventions may contribute to improving the $\mathrm{QOL}$ of maintenance hemodialysis patients who experiences LUTS.

Keywords: Hemodialysis, Lower urinary tract symptoms, Quality of life, Sleep disorders, Validated questionnaires

\section{Background}

Improvement in the quality of life (QOL) of maintenance hemodialysis (MHD) patients is important because poor QOL correlates with mortality and poor adherence to hemodialysis [1-3]. In particular, nighttime waking, declining behavioral desire, and mental instability are reported as representative poor QOL in MHD patients [1-3]. As a cause of these, uremic toxin such as creatinine, inflammatory substances such as C-reactive protein (CRP) and interleukin-6 (IL-6), anemia, and intact parathyroid hormone

\footnotetext{
* Correspondence: chyusuke@oita-u.ac.jp

${ }^{1}$ Department of Urology, Faculty of Medicine, Oita University, Yufu, Japan

${ }^{2}$ Department of Surgery, University of California, San Francisco, CA, USA

Full list of author information is available at the end of the article
}

(iPTH) have been reported [1-3], but the cause is still unknown.

The effects of lower urinary tract symptoms (LUTS), such as nocturia, urinary urgency, frequency, and incontinence, or dysuria attributable to overactive bladder $(\mathrm{OAB})$ and benign prostatic hypertrophy $(\mathrm{BPH})$, can also cause nighttime waking, declining behavioral desire, and mental instability $[4,5]$. The symptoms of $\mathrm{OAB}$ and $\mathrm{BPH}$ are frequently experienced by the elderly $[4,5]$, and $\mathrm{OAB}$ and $\mathrm{BPH}$ can be estimated with validated diseasespecific questionnaires [6-10]. The effect of LUTS on QOL can also be evaluated by a validated questionnaire [11-13]. With aging, and as the duration of their dependence on dialysis becomes longer, MHD patients 
experience decreased urine volume and lose the need to urinate $[14,15]$. There is a possibility that LUTS as QOL disease exists in MHD patients, but there are no reports on LUTS and their effects on QOL in MHD patients. We report an observational study that used validated questionnaires to evaluate LUTS in a group of MHD patients.

\section{Methods}

This study was approved by the Ethics Committee of Nankai Medical Center (No. NH-H25-002) and was performed following the ethical guidelines of the Declaration of Helsinki. All patients gave their written informed consent before enrolling in the study. Informed consent has been obtained from the subjects (or their guardians). Eligible patients were $\geq 40$ years of age $[4,5]$, were receiving MHD three times a week at the Nankai Medical Center, were in stable condition, and were able to agree in writing to participate in this study and to complete the questionnaires. Patients with a history of $\mathrm{OAB}$ or $\mathrm{BPH}$ treatment, kidney transplantation, prostate or bladder cancer, or psychiatric disorder, or a prostate-specific antigen (PSA) level $>4.0 \mathrm{ng} / \mathrm{mL}$, were excluded. Validated Japanese versions of the Overactive Bladder Symptom Score (OABSS) and International Prostate Symptom Score (IPSS) disease-specific, self-administered questionnaires were used to evaluate $O A B$ and BPH [6-10]. The OABSS symptom assessment questionnaire consists of four questions. The possible score ranges from 0 to 15 , with higher scores indicating higher symptom severity [6-8]. IPSS includes seven questions. The possible score ranges from 0 to 35, with higher scores indicating higher symptom severity [6]. King's Health Questionnaire (KHQ) is a self-administered questionnaire for evaluating the QOL of LUTS patients and has been validated in Japanese [11]. It measures the impact of urination symptoms on eight domains: general health perceptions, life, role limitations, physical/social limitations, personal relationships, emotions, sleep/energy, and incontinence severity. The responses in each domain are scored from 0 to 100 , with higher scores indicating greater impairment of QOL [11-13]. An expert nurse and urologist instructed the patients on how to complete the questionnaires. The OABSS, IPSS, and KHQ results and patient clinical data were collected at regularly scheduled clinic visits. Blood samples were collected just before MHD for assays of hemoglobin, albumin, CRP, IL-6, creatinine, and iPTH and PSA in men. Men were evaluated with OABSS, IPSS, and KHQ. Women were evaluated with OABSS and KHQ. Those with urgency scores $\geq 2$ and a total OABSS $\geq 3$ were considered to have OAB [6-8], those with a total IPSS $\geq 8$ were considered to have $\mathrm{BPH}$ [6], and those with $\mathrm{OAB}$ or $\mathrm{BPH}$ were included in the LUTS group. The clinical data and KHQ scores of the LUTS and non-LUTS groups were compared.

\section{Statistical analysis}

Continuous variables were compared with the MannWhitney $U$ test and the Student's $t$ test. Univariate analysis and multivariate analysis of categorical variables were performed using single regression and stepwise multiple regression analysis. Statistical analysis was performed with EZR(Easy-R) software for medical statistics (Saitama Medical Center, Jichi Medical University), which is a graphical user interface for $\mathrm{R}$ commander version 1.6-3 (The $\mathrm{R}$ Foundation for Statistical Computing, version 2.13.0) [16]. The level of statistical significance was a $p$ value $<0.05$.

\section{Results}

This study included 68 men and 63 women with a mean age (standard deviation) of 69.1 (11.3) years and a mean hemodialysis period of 98.1 (95.4) months. Six men and three women had OAB (6.9\%), 13 patients (19.1\%) had $\mathrm{BPH}$, and two of the six men with $\mathrm{OAB}$ also had $\mathrm{BPH}$. There were no significant differences in the age or clinical characteristics of the 20 patients with LUTS (LUTSg) and the 111 without LUTS (non-LUTSg). The HD period was significantly shorter $(p<0.01)$ in LUTSg than non-LUTSg. The mean KHQ score was significantly higher $(p<0.01)$ in the LUTSg than in the non-LUTSg. The impact on life $(p<$ $0.01)$, role limitations $(p=0.01)$, emotions $(p<0.01)$, sleep/ energy $(p<0.01)$, and incontinence severity $(p=0.01)$ domain scores were higher in LUTSg, but the differences in the general health perception, physical limitation, and social limitation domain scores of the two groups were not significant (Table 1). As shown in Table 2, single regression analysis and stepwise multiple regression analysis showed that LUTS was independently associated with the total KHQ score (correlation coefficient 0.292; 95\% confidence interval 20.36-89.47; $p<0.01)$.

\section{Discussion}

The disease-specific questionnaires confirmed that MHD patients experienced LUTS. The KHQ scores confirmed that LUTS impair the QOL in MHD patients and had a negative impact on five domains: life, role limitations, emotions, sleep/energy, and incontinence severity. The correlation coefficient 0.292 is regarded as the weak correlation between LUTS and KHQ score. This seems to be related to the fact that there were no significant differences between the two groups in the other three domains. However, the five domains are closely related to representative factors that impair the QOL of MHD patients, such as nighttime waking, deciding behavioral desire, and mental instability [1-3]. Therefore, LUTS should be recognized as an important QOL disease in MHD patients same as the general population. 
Table 1 Characteristics of overall participants and of patients in the two groups being studied

\begin{tabular}{|c|c|c|c|c|}
\hline Variable & Overall $(n=131)$ & LUTS group $(n=20)$ & Non-LUTS group $(n=111)$ & Significance $(p)$ \\
\hline Male/female & $68 / 63$ & $17 / 3$ & $51 / 60$ & 0.001 \\
\hline Age(years) & $69.1 \pm 11.3$ & $70.45 \pm 10.39$ & $68.79 \pm 11.38$ & 0.55 \\
\hline Duration on hemodialysis (months) & $98.1 \pm 95.4$ & $46.65 \pm 46.65$ & $107.11 \pm 99.1$ & 0.009 \\
\hline Total OABSS & $0.74 \pm 1.77$ & $3.35 \pm 3.4$ & $0.31 \pm 0.61$ & $<0.001$ \\
\hline Total IPSS & $4.19 \pm 5.79$ & $11.59 \pm 6.87$ & $1.85 \pm 2.31$ & $<0.001$ \\
\hline \multicolumn{5}{|l|}{ KHQ domains } \\
\hline General health perceptions & $40.6 \pm 22.3$ & $42.5 \pm 16.0$ & $40.40 \pm 23.20$ & 0.7 \\
\hline Impact on life & $7.54 \pm 20.0$ & $20.0 \pm 28.64$ & $5.25 \pm 16.94$ & 0.002 \\
\hline Role limitations & $2.50 \pm 9.91$ & $8.1 \pm 13.09$ & $1.76 \pm 9.28$ & 0.01 \\
\hline Physical limitations & $0.98 \pm 5.15$ & $1.6 \pm 6.97$ & $0.87 \pm 4.76$ & 0.56 \\
\hline Social limitations & $1.30 \pm 9.38$ & $0.0 \pm 0.0$ & $1.517 \pm 10.16$ & 0.50 \\
\hline Personal relationships & $2.76 \pm 13.1$ & $0.0 \pm 0.0$ & $3.25 \pm 14.16$ & 0.35 \\
\hline Emotions & $6.34 \pm 16.0$ & $17.6 \pm 26.81$ & $4.26 \pm 12.01$ & $<0.001$ \\
\hline Sleep/energy & $7.20 \pm 16.1$ & $20.8 \pm 25.85$ & $4.71 \pm 12.03$ & $<0.001$ \\
\hline Incontinence severity & $12.2 \pm 13.2$ & $19.15 \pm 13.54$ & $11.02 \pm 12.75$ & 0.01 \\
\hline Total KHQ score & $77.8 \pm 67.2$ & $120.4 \pm 87.79$ & $70.20 \pm 59.65$ & 0.001 \\
\hline \multicolumn{5}{|l|}{ Clinical data } \\
\hline Hemoglobin (g/dL) & $10.22 \pm 1.62$ & $10.38 \pm 1.68$ & $10.19 \pm 1.26$ & 0.64 \\
\hline Serum albumin (g/L) & $3.481 \pm 0.402$ & $33.55 \pm 2.99$ & $35.03 \pm 4.15$ & 0.13 \\
\hline Serum CRP (mg/L) & $0.3428 \pm 0.6495$ & $28.31 \pm 45.30$ & $34.36 \pm 68.10$ & 0.70 \\
\hline Serum IL-6 (pg/mL) & $10.803 \pm 15.564$ & $8.323 \pm 6.302$ & $11.36 \pm 16.67$ & 0.43 \\
\hline Serum creatinine (mg/L) & $10.426 \pm 2.087$ & $103.43 \pm 17.44$ & $104.4 \pm 21.58$ & 0.85 \\
\hline Serum calcium (mg/L) & $9.305 \pm 0.916$ & $93.20 \pm 7.85$ & $93.02 \pm 9.44$ & 0.94 \\
\hline Serum phosphate (mg/L) & $5.140 \pm 1.705$ & $46.1 \pm 14.86$ & $52.34 \pm 17.38$ & 0.133 \\
\hline Serum intact PTH (pg/mL) & $228.345 \pm 203.34$ & $227.23 \pm 254.87$ & $228.55 \pm 194.52$ & 0.978 \\
\hline
\end{tabular}

A recent epidemiological survey found that the percentage of men and women with LUTS increased with age and was $8-12 \%$ in the $40 \mathrm{~s}, 50-60 \%$ in the $60 \mathrm{~s}$, and $80 \%$ in the 80 s $[4,5]$. The frequency of LUTS in this study was approximately $15.3 \%$, which is very low compared to the frequency of the general population. LUTS related to urine storage, such as urgency, frequency, nocturia, and urge incontinence, interfere with daily living and impair QOL [4-13].

Subjective symptoms and QOL evaluations may be more informative in routine clinical examinations than in objective examinations such as urinary flowmetry, residual urine volume, and cystometric pressure measurement for determining the impact of LUTS [4-13]. The OABSS and IPSS are determined by the responses to questionnaires validated for $\mathrm{OAB}$ and $\mathrm{BPH}$, which are the primary diseases underlying LUTS [4-13]. KHQ is a validated questionnaire used in clinical practice to evaluate the QOL of patients with LUTS [11-13]. The questionnaires easily screen patients for $\mathrm{OAB}$ and $\mathrm{BPH}$ symptoms and QOL and are tools frequently used in epidemiological studies and assessments of therapeutic effect [4-13]. However, there have been no previous surveys of the incidence of LUTS in MHD patients.

Residual renal function decreases with the duration of the dialysis requirement, and MHD patients ultimately progress to oliguria, anuria, and loss of urination [15, 16]. Until now, it was not known whether LUTS occurred in MHD patients and whether it impacted QOL. This study confirmed that some MHD patients experience LUTS. Desmopressin has been reported to reduce the urine volume in patients with LUTS who did not respond to anticholinergic drugs, which were the first drug class used in LUTS therapy [17]. It is thus reasonable to assume that the urine volume produced in patients with a short dialysis duration can be linked to the development of LUTS, as seen in this study.

Approximately 45\% of MHD patients experience sleep disorders, and $90 \%$ of the reported disorders are nighttime waking $[1-3,8-20]$. Sleep disorders have been linked to decreased survival, perceived decrease of health-related QOL, and poor adherence to hemodialysis 
Table 2 Univariate and multivariate analysis between the total KHQ score and clinical and laboratory parameters

\begin{tabular}{|c|c|c|c|c|c|}
\hline Explanatory variable & Regression coefficient & Standard error & Correlation coefficient & 95\% confidence interval & $p$ value \\
\hline \multicolumn{6}{|l|}{ Single regression analysis } \\
\hline Sex & 1.444 & 11.74 & 0.010 & -21.78 to 24.67 & 0.902 \\
\hline Age(years) & -0.715 & 0.519 & -0.119 & -1.743 to 0.313 & 0.171 \\
\hline Duration on hemodialysis (months) & -0.014 & 0.063 & -0.020 & -0.139 to 0.109 & 0.814 \\
\hline LUTS & 50.59 & 15.80 & 0.269 & 19.325 to 81.86 & 0.002 \\
\hline \multicolumn{6}{|l|}{ Clinical data } \\
\hline Hemoglobin (g/dL) & 1.821 & 3.62 & 0.043 & -5.532 to 8.995 & 0.616 \\
\hline Serum albumin (g/L) & 1.921 & 1.45 & 0.011 & -26.93 to 30.77 & 0.895 \\
\hline Serum CRP (mg/L) & -3.425 & 9.027 & -0.033 & -21.28 to 14.43 & 0.704 \\
\hline Serum IL-6 (pg/mL) & -0.169 & 0.379 & -0.039 & -0.919 to 0.580 & 0.655 \\
\hline Serum creatinine (mg/L) & 2.724 & 2.799 & 0.084 & -2.813 to 8.263 & 0.332 \\
\hline Serum calcium (mg/L) & -1.004 & 6.403 & -0.013 & -13.67 to 11.66 & 0.875 \\
\hline Serum phosphate (mg/L) & -0.236 & 3.439 & -0.050 & -7.017 to 6.590 & 0.950 \\
\hline Serum intact PTH (pg/mL) & 0.055 & 0.028 & 0.169 & -0.003 to 0.112 & 0.051 \\
\hline \multicolumn{6}{|l|}{ Stepwise multiple regression analysis } \\
\hline LUTS & 54.91 & 17.45 & 0.292 & 20.36 to 89.47 & 0.002 \\
\hline Serum intact PTH (pg/mL) & 0.060 & 0.031 & 0.171 & -0.002 to 0.122 & 0.059 \\
\hline
\end{tabular}

treatment $[1-3,8-20]$. The impacts of clinical data including systemic inflammation, dialysis time, and dialysis cycle on QOL and on sleep disorders have been reported [18-20]. The clinical characteristics of the LUTS and non-LUTS patients in this study were not significantly different and were in line with those reported in previous studies. However, a specific questionnaire, KHQ, identified sleep disorders associated with LUTS as affecting QOL. It is very important to evaluate LUTS as a cause of sleep disorder in MHD patients and to consider whether their sleep disorders might be improved by urological interventions. QOL improvement would be expected to directly improve both adherence to treatment and the survival of MHD patients [1-3, 18-20]. MHD patients with perceived problems of daily living may experience feelings of depression, unstable behavior, or insomnia [1-3, 18-20]. It is important that hemodialysis caregivers understand that LUTS may be involved and may cause a decrease in QOL and that a urological intervention may be effective.

The study limitations included symptom diagnosis by disease-specific questionnaires. The diagnostic accuracy of LUTS would have been increased by objective examinations to estimate prostate weight, measure daily urine volume, and perform urinalysis. Moreover, this was a cross-sectional study including a small number of patients. The clinical value of the OABSS, IPSS, and KHQ assessments can be increased by further evaluation in larger studies including patients with longer dialysis histories. This study found that MHD patients experience LUTS that may impair their QOL. Validated questionnaires are convenient to screen MHD patients who experience LUTS and assess the influence of LUTS on QOL. Urological interventions may contribute to the improvement in the QOL of MHD patients who experience LUTS.

\section{Conclusions}

LUTS exists in MHD patients and impairs the QOL of them assessed with validated questionnaires. Urological interventions may contribute to improving the QOL of maintenance hemodialysis patients who experiences LUTS.

\section{Abbreviations}

$\mathrm{BPH}$ : Benign prostatic hypertrophy; CRP: C-reactive protein; IL-6: Interleukin-6; IPSS: International Prostate Symptom Score; iPTH: Intact parathyroid hormone; KHQ: King's Health Questionnaire; LUTS: Lower urinary tract symptoms; MHD: Maintenance hemodialysis; OAB: Overactive bladder; OABSS: Overactive Bladder Symptom Score; PSA: Prostate-specific antigen; QOL: Quality of life

\section{Acknowledgements}

Not applicable.

\section{Authors' contributions}

TA planned and conducted the study, analyzed the data, and drafted the manuscript. TA and TK recruited the patients, collected the patient samples, and conducted the sample analysis. TS and HM supervised the study and drafted the manuscript. All authors gave their final approval for the final version of the manuscript prior to submission.

\section{Funding}

Not applicable.

\section{Availability of data and materials}

All data generated or analyzed during this study are included in the published article. 


\section{Ethics approval and consent to participate}

The study protocol was approved by the ethics committee at Nankai Medical Center (IRB approval number NH-H25-002) as an epidemiological study conducted on validated questionnaires and patient blood samples. Written consent for enrollment and publication was obtained from patients for the extended use of validated questionnaires and patient blood samples obtained for usual clinical practice. Patients were informed about the details of the study by a documented explanation form and were given the right to withdraw consent after enrollment.

\section{Consent for publication}

Not applicable.

\section{Competing interests}

The authors declare that they have no competing interests.

\section{Author details}

'Department of Urology, Faculty of Medicine, Oita University, Yufu, Japan. 2Department of Surgery, University of California, San Francisco, CA, USA.

${ }^{3}$ Hemodialysis Center, Nankai Medical Center, Saiki, Japan.

Received: 25 March 2019 Accepted: 19 August 2019

Published online: 30 August 2019

\section{References}

1. D'Onofrio G, Simeoni M, Rizza P, et al. Quality of life, clinical outcome, personality and coping in chronic hemodialysis patients. Ren Fail. 2017; 39:45-53.

2. Anand S, Johansen KL, Grimes B, et al. Physical activity and self-reported symptoms of insomnia, restless legs syndrome, and depression; the comprehensive dialysis study. Hemodial Int. 2013;17:50-8.

3. Wang $R$, Tang C, Chen $X$, et al. Poor sleep and reduced quality of life were associated with symptom distress in patients receiving maintenance hemodialysis. Health and Quality of Life Outcomes. 2016;14:125-32.

4. Parsons JK, Wilt TJ, Wang PY, et al. Progression of lower urinary tract symptoms among older men: a community based study. J Urol. 2010;183: 1915-20.

5. Coyne KS, Sexton CC, Bell JA, et al. The prevalence of lower urinary tract symptoms (LUTS) and overactive bladder (OAB) by racial/ethnic group and age: results from OAB-POLL. Neurourol Urodyn. 2013;32:230-7.

6. Chuang F-C, Hsiao S-M, Kuo H-C. The overactive bladder symptom score, international prostate symptom score-storage sub score, and urgency severity score in patients with overactive bladder and hypersensitive bladder: which scoring system is best? Int Neurourol J. 2018;22:99-106.

7. Nozawa Y, Kato D, Tabuchi H, Kuroishi K. Safety and effectiveness of mirabegron in patients with overactive bladder in a real-world clinical setting: a Japanese post-marketing study. LUTS. 2018;10:122-30.

8. Lee K-S, Yoo TK, Liao L, et al. Association of lower urinary tract symptoms and $O A B$ severity with quality of life and mental health in China, Taiwan and South Korea: results from a cross-sectional, population-based study. BMC Urology. 2017;17:108-17.

9. Martin S, Vincent A, Taylor AW, et al. Lower urinary tract symptoms, depression, anxiety and systemic inflammatory factors in men: a populationbased cohort study. PLOS ONE. 2015. https://doi.org/10.1371/journal.pone. 0137903.

10. Kwon BE, Kim GY, Son YJ, Roh YS, You MA. Quality of life of women with urinary incontinence: a systematic literature review. Int Neurourol J. 2010;14:133-8.

11. Okamura K, Usami T, Nagahama K, Maruyama S, Mizuta E. "Quality of life" assessment of urination in elderly Japanese men and women with some medical problems using International Prostate Symptom Score and King's Health Questionnaire. Eur Urol. 2002;41:411-9.

12. Reese PR, Pleil AM, Okano GJ, Kelleher CJ. Multinational study of reliability and validity of the King's Health Questionnaire in patients with overactive bladder. Qual Life Res. 2003;12:427-42.

13. Uemura S, Homma Y. Reliability and validity of King's Health Questionnaire in patients with symptoms of overactive bladder with urge incontinence in Japan. Neurourol Urodyn. 2004;23:94-100.

14. Mokoli VM, Sumaili EK, Lepira FB, et al. Factors associated with residual urine volume preservation in patients undergoing hemodialysis for end-stage kidney disease in Kinshasa. BMC Nephrology. 2018;19:68-76.
15. Mokoli VM, Sumaili EK, Lepira FB, et al. Impact of residual urine volume decline on the survival of chronic hemodialysis patients in Kinshasa. BMC Nephrology. 2016;17:182-91.

16. Kanda Y. Investigation of the freely available easy-to-use software 'EZR' for medical statistics. Bone Marrow Transplantation. 2013;48:452-8.

17. Giannitsas K, Athanasopoulos A. Desmopressin for the treatment of female storage lower urinary tract symptoms. World J Obstet Gynecol. 2014;3:7-13.

18. Hamzi MA, Hassani K, Asseraji M, El Kabbaj D. Insomnia in hemodialysis patients: a multicenter study from Morocco. Saudi J Kidney Dis Transpl. 2017;28:1112-8

19. Sabbatini M, Minale B, Crispo A, et al. Insomnia in maintenance hemodialysis patients. Nephrol Dial Transplant. 2002;17:852-6.

20. Maung SC, El Sara A, Chapman C, Cohen D, Cukor D. Sleep disorders and chronic kidney disease. World J Nephrol. 2016;5:224-32.

\section{Publisher's Note}

Springer Nature remains neutral with regard to jurisdictional claims in published maps and institutional affiliations.
Ready to submit your research? Choose BMC and benefit from:

- fast, convenient online submission

- thorough peer review by experienced researchers in your field

- rapid publication on acceptance

- support for research data, including large and complex data types

- gold Open Access which fosters wider collaboration and increased citations

- maximum visibility for your research: over $100 \mathrm{M}$ website views per year

At BMC, research is always in progress.

Learn more biomedcentral.com/submissions 